\title{
Efeito de Herbicidas sobre Plantas de Brachiaria plantaginea SUBMETIDAS A ESTRESSE HÍdRICO ${ }^{1}$
}

\author{
Effect of Herbicides on Brachiaria plantaginea Plants Submitted to Water Stress
}

\author{
PEREIRA, M.R.R. ${ }^{2}$, MARTINS, D. ${ }^{3}$, SILVA, J.I.C. ${ }^{4}$, RODRIGUES-COSTA, A.C.P. ${ }^{5}$ e \\ KLAR, A.E. ${ }^{6}$
}

\begin{abstract}
RESUMO - Este trabalho objetivou avaliar a eficiência de controle de herbicidas inibidores da ACCase aplicados em pós-emergência em plantas de Brachiaria plantaginea submetidas a diferentes teores de água no solo. O estudo foi conduzido em casa de vegetação. $O$ delineamento experimental foi o inteiramente casualizado, com quatro repetições, utilizando-se o esquema fatorial $9 \times 4$, sendo a combinação de três manejos hídricos $(-0,03$, -0,07 e -1,5 MPa) com três herbicidas (fluazifop-p-butil, haloxyfop-methyl e sethoxydim + óleo mineral Assist) e quatro doses destes (100, 50, 25 e 0\% da dose recomendada). A aplicação dos herbicidas foi realizada em dois estádios vegetativos das plantas: 4-6 folhas e 2-3 perfilhos. As avaliações visuais de fitointoxicação das plantas de B. plantaginea foram realizadas aos 3 , 7 e 14 dias após a aplicação, sendo determinada a massa seca das plantas no final do experimento. A eficiência de controle foi menor em plantas mantidas em potencial de água no solo de -1,5 MPa (manejo hídrico de 8\%), independentemente do herbicida utilizado, nos dois estádios de aplicação, com exceção do herbicida haloxyfop-methyl aplicado no estádio de 2-3 perfilhos. Não houve diferenças de controle entre as aplicações com 100 e $50 \%$ da dose recomendada dos herbicidas nas plantas no estádio de 4-6 folhas, independentemente do manejo hídrico.
\end{abstract}

Palavras-chave: controle químico, estresse hídrico, planta daninha, capim-marmelada.

\begin{abstract}
This work aimed to evaluate the efficiency in controlling ACCase inhibiting-herbicides applied in post-emergence on Brachiaria plantaginea, submitted to different soil water contents. The study was carried out under greenhouse conditions. The experiment was arranged in a completely randomized block design with four replications, consisting of a $9 \times 4$ factorial, with the combination of three water management systems (-0.03, -0.07 and -1.5 MPa) and three herbicides (fluazifop-pbutyl, haloxyfop-methyl and sethoxydim + oil Assist) and four doses of these herbicides (100, 50, 25 and $0 \%$ of the recommended dose). Herbicide was applyed at two vegetative stages of the plants, 4-6 leaves and 2-3 tillers. The visual assessments of plant toxicity were performed at 3, 7 and 14 days after application, with the dry matter of the plants being determined at the end of the experiment. Control efficiency was lower in the plants grown at soil water potential of $-1.5 \mathrm{MPa}$ (water management $8 \%$ ), regardless of the herbicide used at the two stages of implementation, with the exception of the herbicide haloxyfop-methyl applied at the 2-3 tillers. No differences were found between the control applications with 100 and $50 \%$ of the recommended herbicide doses on plants at the 4-6 leaf stage, regardless of type of water management.
\end{abstract}

Keywords: chemical control, water stress, weed, Brachiaria plantaginea.

Recebido para publicação em 15.7.2010 e na forma revisada em 17.12.2010.

${ }^{2}$ Professora, Dra., Assistente, Faculdade de Tecnologia do Estado de São Paulo - FATEC, 18304-750 Capão Bonito-SP, $<$ mariarenata10@hotmail.com>; ${ }^{3}$ Professor Adjunto, Dep. de Produção Vegetal, Faculdade de Ciências Agronômicas - FCA/UNESP, Caixa Postal 237, 18603-970 Botucatu-SP; ${ }^{4}$ Doutor em Agronomia/Agricultura; ${ }^{5}$ Professora, Colaboradora, Universidade Estadual do Oeste do Paraná - UNIOESTE, Caixa Postal 1008, Marechal Cândido Rondon-PR; ${ }^{6}$ Professor Titular, Dep. de Engenharia Rural, FCA/UNESP, Botucatu-SP. 


\section{INTRODUÇÃO}

Das plantas daninhas consideradas importantes em diferentes culturas agrícolas, a Brachiaria plantaginea é a gramínea de maior ocorrência na região centro-sul do Brasil, ocorrendo também em diferentes sistemas produtivos de países como Argentina e Paraguai (Kissmann, 1997; Lorenzi, 2000). Essa espécie é bastante competitiva, principalmente em culturas anuais, como soja e milho, provocando prejuízos consideráveis ao rendimento e à qualidade da produção (Spacer \& Vidal, 2000).

Os herbicidas fluazifop-p-butil, haloxyfopmethyl e sethoxydim, os quais são recomendados para o controle de $B$. plantaginea, entre outras gramíneas, pertencem ao grupo dos inibidores da ACCase. O modo de ação primário deles consiste na inibição da síntese de ácidos graxos, ao inibirem a enzima acetil coenzimaA carboxilase (ACCase). Esta enzima regula uma reação-chave no início da biossintese de lipídeos necessária para síntese e manutenção de membranas, e não na produção de aminoácidos (converte o acetil coenzima-A em malonil coenzima-A). A ACCase presente em gramineas é, geralmente, sensível à inibição por esses herbicidas (Rodrigues, 1994).

De acordo com Abbott \& Sterling (2006), a eficácia de um herbicida pode ser comprometida pela menor disponibilidade de água no ambiente no qual a planta daninha está se desenvolvendo, influenciando na sua absorção, translocação e metabolismo. Estudos citados por Deuber (1992) mostram que a absorção dos herbicidas é limitada pela quantidade que atravessa a cutícula da folha, e esta é influenciada pelas condições ambientais, como umidade no solo, bem como pelas características do herbicida e pelo estádio de desenvolvimento das plantas daninhas.

Alguns pesquisadores comprovaram efeitos reduzidos dos herbicidas em plantas submetidas a estresse hídrico, como, por exemplo: imazametabenze em Avena fatua (Xie et al., 1996); glyphosate em Asclepias syriaca (Waldecker \& Wyse, 1985); e haloxyfop-methyl em Sorghum halapense (Peregoy et al., 1990) e em Digitaria sanguinales, sendo o controle desta de $92 \%$ em plantas sem restrição hídrica e de $8 \%$ para aquelas submetidas a estresse hídrico (Dickson et al.,1990). De acordo com pesquisas realizadas por Boydston (1990), o menor controle de Setaria viridis foi verificado com sethoxydim quando esta planta foi submetida a um menor teor de água no solo. Da mesma forma, Kells et al. (1984) verificaram menor absorção foliar de fluazifop-p-butil devido à baixa umidade no solo.

Outra questão relevante a ser estudada é a utilização de doses elevadas de herbicidas, o que vem sendo intensamente questionado nos últimos anos, em razão dos possiveis danos causados ao agroecossistema, do custo elevado e do aumento da pressão de biótipos resistentes a herbicidas. Entretanto, as doses recomendadas de alguns herbicidas podem ser reduzidas sem prejuízo para o controle das plantas daninhas e sem afetar o rendimento da cultura, podendo ser associadas a práticas de manejo, o que possibilita manter o controle de plantas daninhas em niveis satisfatórios, sendo mais ecologicamente corretas e economicamente viáveis (Fleck et al., 1997; Pires et al., 2001).

Dessa forma, a importância de Brachiaria plantaginea - bem como de outras gramineas infestantes em sistemas produtivos - justifica o desenvolvimento de metodologias eficientes, simples e economicamente viáveis para avaliações mais rigorosas da eficiência de herbicidas em diferentes condições ambientais.

O objetivo deste estudo foi avaliar a eficiência de controle de herbicidas inibidores da ACCase aplicados em pós-emergência em plantas de $B$. plantaginea submetidas a estresse hídrico.

\section{MATERIAL E MÉTODOS}

O experimento foi instalado e conduzido no Departamento de Produção Vegetal, Setor Agricultura, da Faculdade de Ciências Agronômicas, UNESP, no município de Botucatu - São Paulo, cujas coordenadas geográficas são $22^{\circ} 51^{\prime} 03^{\prime}$ de latitude sul e 48 $25^{\prime} 37^{\prime \prime}$ de longitude oeste, com altitude de $786 \mathrm{~m}$.

Os estudos foram instalados nos meses de agosto a novembro de 2008, com as seguintes caracterizações climáticas nesse período dentro da casa de vegetação (média): temperatura mínima de $17,1{ }^{\circ} \mathrm{C}$, temperatura máxima de $25{ }^{\circ} \mathrm{C}$, umidade relativa do ar de $54,6 \%$ e 
evapotranspiração de $4,16 \mathrm{~mm}$ mês ${ }^{-1}$, a qual foi monitorada diariamente por meio de um tanque classe A.

A espécie utilizada foi Brachiaria plantaginea, cultivada em vasos plásticos de $2 \mathrm{~L}$, mantidos em casa de vegetação. Foi realizada adubação do solo de acordo com a análise química (Tabela 1). A descrição textural do solo foi classificada como média pela análise granulométrica $(65,6 \%$ de areia, $6,7 \%$ de silte e $27,7 \%$ de argila).

O solo, antes do plantio, foi seco ao ar, revolvido duas vezes por semana até peso constante, com o objetivo de reduzir a um mínimo de umidade no solo, que foi de $3 \%$, para realização dos cálculos de reposição de água, de acordo com a curva de retenção. Para obtenção da curva de retenção de água foi utilizada a placa de pressão de Richards (Klar, 1984). A partir dos resultados da curva de retenção, foram estabelecidos três potenciais mínimos de água ( $\Psi$ s): $-0,03,-0,07$ e - 1,5 MPa, sendo 13, 10 e $8 \%$ de umidade do solo, respectivamente, compondo os manejos hídricos, avaliados por meio de pesagem dos vasos. As relações entre os potenciais de água e a porcentagem de umidade do solo encontram-se na Tabela 3. Ao atingir valores próximos do potencial definido para cada tratamento, foi feita reposição da água evapotranspirada até alcançar massa do potencial de água máximo de retenção de água do solo (-0,01 MPa/14\% de umidade do solo). Os manejos hídricos foram iniciados no estádio de desenvolvimento de duas folhas em cada planta.

A aplicação dos herbicidas foi feita em duas fases de desenvolvimento das plantas daninhas (4-6 folhas e 2-3 perfilhos). Foi utilizado um pulverizador costal, equipado com barra de aplicação contendo quatro pontas de pulverização tipo jato plano XR 11002 VS, com consumo de calda de $200 \mathrm{~L} \mathrm{ha}^{-1}$. Os tratamentos foram dispostos em um fatorial $9 \times 4$, sendo o primeiro fator composto por nove combinações: três herbicidas (fluazifop-p-butil, haloxyfopmethyl e sethoxydim) e três manejos hídricos ( $\Psi$ s), e o segundo fator, por quatro doses dos produtos $(0,25,50$ e $100 \%$ da dose recomendada). De acordo com os fabricantes de cada produto, $100 \%$ da dose recomendada de cada herbicida, expressa em gramas de ingrediente ativo por hectare, é de (g i.a. ha $\left.{ }^{-1}\right)$ : sethoxydim: 184, haloxyfop-methyl: 60 e fluazifop-p-butil: 125.

Os efeitos de intoxicação dos tratamentos sobre as plantas foram avaliados visualmente aos 3, 7 e 14 dias após aplicação, dependendo da espécie estudada, por meio de uma escala percentual de notas, na qual 0 consiste em ausência de injúria e 100 em morte das plantas (SBCPD, 1995). Ao final das avaliações, as plantas foram coletadas e secas em estufa de ventilação forçada de ar a $60^{\circ} \mathrm{C}$ até atingir peso constante; em seguida, foi determinada a massa seca em g por planta.

O delineamento experimental utilizado nos estudos foi o inteiramente casualizado com quatro repetições. Os resultados de fitointoxição foram submetidos à análise de variância pelo teste $\mathrm{F}$, e os dados de massa seca de plantas, aos modelos de regressão linear e polinomial, sendo as médias dos tratamentos comparadas pelo teste de Tukey a $5 \%$ de probabilidade.

\section{RESULTADOS E DISCUSSÃO}

Os resultados da eficiência de controle da aplicação de alguns herbicidas e doses sobre plantas de $B$. plantaginea, no estádio de 4-6 folhas, mantidas em diferentes umidades do solo, podem ser observados na Tabela 2. Aos três dias após aplicação dos herbicidas (DAA), todos os tratamentos apresentavam poucos sintomas causados por eles, sendo maiores com a aplicação de fluazifop-p-butil e sethoxydim, com $100 \%$ da dose recomendada, em plantas sem estresse hídrico (manejo

Tabela 1 - Análise química do solo utilizado no estudo. Botucatu-SP, 2008

\begin{tabular}{|c|c|c|c|c|c|c|c|c|c|c|}
\hline $\mathrm{pH}$ & $\mathrm{MO}$ & $\mathrm{P}$ resina & $\mathrm{H}+\mathrm{Al}$ & $\mathrm{K}$ & $\mathrm{Ca}$ & $\mathrm{Mg}$ & $\mathrm{SB}$ & $\mathrm{CTC}$ & $\mathrm{V}$ \\
\hline$\left(\mathrm{CaCl}_{2}\right)$ & $\left(\mathrm{g} \mathrm{dm}^{-3}\right)$ & $\left(\mathrm{mg} \mathrm{dm}^{-3}\right)$ & \multicolumn{7}{|c|}{$\left(\mathrm{mmol} \mathrm{dm}^{3}\right)$} & $(\%)$ \\
\hline 4,6 & 7 & 3 & 22 & 0,2 & 2 & 2 & 4 & 26 & 15 \\
\hline
\end{tabular}


Tabela 2 - Porcentagem de controle em plantas de B. plantaginea no estádio de 4-6 folhas submetidas a diferentes manejos hídricos, três dias após aplicação de herbicidas. Botucatu-SP, 2008

\begin{tabular}{|c|c|c|c|c|c|}
\hline \multicolumn{2}{|c|}{ Tratamento } & \multicolumn{4}{|c|}{$\%$ da dose de herbicida } \\
\hline Herbicida & Manejo hídrico (MPa) & 0 & 25 & 50 & 100 \\
\hline \multirow{3}{*}{ sethoxydim } & $-0,03$ & 0,00 a C & 7,25 bcd B & $9,00 \mathrm{bc} \mathrm{B}$ & $15,75 \mathrm{ab} A$ \\
\hline & $-0,07$ & 0,00 a $\mathrm{C}$ & 7,25 bcd B & 8,50 bcd $A B$ & $11,00 \mathrm{c} \mathrm{A}$ \\
\hline & $-1,50$ & 0,00 a B & $3,75 \mathrm{~d} \mathrm{~A}$ & $4,50 \mathrm{dA}$ & $5,25 \mathrm{dA}$ \\
\hline \multirow{3}{*}{ haloxyfop-methyl } & $-0,03$ & 0,00 a B & $10,00 \mathrm{ab} \mathrm{A}$ & $11,00 \mathrm{~b} \mathrm{~A}$ & $11,75 \mathrm{bc} \mathrm{A}$ \\
\hline & $-0,07$ & $0,00 \mathrm{a} \mathrm{B}$ & $8,25 \mathrm{abc} A$ & $9,00 \mathrm{bc} \mathrm{A}$ & $10,50 \mathrm{c} \mathrm{A}$ \\
\hline & $-1,50$ & 0,00 a B & $3,25 \mathrm{~d} A B$ & $5,50 \mathrm{~cd} \mathrm{~A}$ & $5,25 \mathrm{~d} A$ \\
\hline \multirow{3}{*}{ fluazifop-p-butil } & $-0,03$ & 0,00 a C & 12,50 a B & 16,75 a A & 19,50 a $\mathrm{A}$ \\
\hline & $-0,07$ & 0,00 a B & 8,25 abc $A$ & $10,75 \mathrm{~b} \mathrm{~A}$ & $9,25 \mathrm{~cd} \mathrm{~A}$ \\
\hline & $-1,50$ & 0,00 a B & $4,75 \mathrm{~cd} \mathrm{~A}$ & $5,75 \mathrm{~cd} \mathrm{~A}$ & $6,00 \mathrm{~d} \mathrm{~A}$ \\
\hline $\mathrm{F}_{\text {tratamento }}(\mathrm{T})$ & \multicolumn{5}{|c|}{$33,779^{* *}$} \\
\hline $\mathrm{F}_{\text {dose }}(\mathrm{D})$ & \multicolumn{5}{|c|}{$210,695^{* *}$} \\
\hline $\mathrm{F}(\mathrm{T}) \times(\mathrm{D})$ & \multicolumn{5}{|c|}{$5,477^{* *}$} \\
\hline $\mathrm{CV}(\%)$ & \multicolumn{5}{|c|}{28,7} \\
\hline
\end{tabular}

Médias seguidas de mesma letra, minúscula na coluna e maiúscula na linha, não diferem entre si pelo teste de Tukey ( $\mathrm{p}>0,05)$; ** Significativo a $1 \%$ de probabilidade.

hídrico de 13\%). Já os sintomas provocados pelo herbicida haloxyfop-methyl foram semelhantes em plantas submetidas aos manejos hídricos de 13 e $10 \%$.

Os sintomas também foram semelhantes entre si independentemente do herbicida em plantas mantidas em solo com $10 \%$ de umidade, alcançando em média 10,5\% de controle, fato também observado nas plantas submetidas a manejo hídrico de $8 \%$, com controle máximo de $6 \%$.

Com a aplicação de 50\% da dose recomendada, os herbicidas sethoxydim e haloxyfop-methyl proporcionaram resultados semelhantes, e os controles foram idênticos com as plantas mantidas nos manejos hídricos de 13 e $10 \%$. O herbicida fluazifop-p-butil teve o mesmo comportamento quando foram utilizados $100 \%$ da dose, obtendo os melhores controles em plantas sem estresse hídrico $(16,75 \%)$. A fitointoxicação foi a mesma para todos os herbicidas em plantas sob restrição hídrica severa (manejo hídrico de $8 \%$ ): em média, $5 \%$. Os resultados do fracionamento da dose recomendada em $25 \%$ foram semelhantes aos da aplicação de $50 \%$ da dose.

Aos 7 DAA, os herbicidas com melhores controles foram o sethoxydim, seguido pelo fluazifop-p-butil, com aplicação de $100 \%$ da dose, nos manejos hídricos de 13 e $10 \%$, não sendo observadas diferenças de controle entre plantas mantidas nesses manejos, atingindo até $46,25 \%$ de injúrias (Tabela 3). O mesmo comportamento foi observado com o uso do herbicida haloxyfop-methyl em menor proporção (controle em média de 50\%).

Em plantas sob restrição hídrica (manejo hídrico de $8 \%$ ), verificaram-se os piores controles, independentemente dos herbicidas testados, sendo em média $60 \%$ menor do que o controle em plantas sem restrição hídrica (manejo hídrico de 13\%).

Não se observaram diferenças dos sintomas com aplicação de 50 e $25 \%$ da dose dos herbicidas, independentemente dos manejos hídricos utilizados, com exceção do uso do sethoxydim em solo a um mínimo de $13 \%$ de umidade e do haloxyfop-methyl e fluazifopp-butil em solo a um mínimo de $8 \%$ de umidade.

Em todas as plantas submetidas ao manejo hídrico de $8 \%$, não houve diferença no controle entre as doses, independentemente do produto aplicado. Pode-se inferir que, em plantas submetidas a maior estresse hídrico, a eficiência de controle é insatisfatória mesmo com o uso da dose recomendada, a qual se iguala aos resultados quando se aplica um quarto da dose. 
Na Tabela 4, observou-se eficiência de controle satisfatória (acima de 97\%) com a aplicação de $100 \%$ da dose recomendada de todos os três herbicidas em plantas submetidas a manejos hídricos de 13 e 10\%. Nas aplicações em plantas mantidas sob estresse hídrico (minimo de $8 \%$ de umidade do solo), os melhores controles foram verificados com o uso dos herbicidas sethoxydim e haloxyfop-methyl, atingindo 85 e $89,5 \%$, respectivamente, e os resultados obtidos com o herbicida fluazifop-pbutil não alcançaram os $80 \%$.

Com o uso de $50 \%$ da dose dos herbicidas, a eficiência de controle foi acima de $90 \%$ nas plantas com manejo hídrico de $13 \%$ de umidade mínima do solo e de acima de $80 \%$ nas aplicações de $25 \%$ da dose, independentemente dos produtos utilizados. Não se observaram diferenças na fitotoxicidade entre as plantas com manejo de 13 e $10 \%$ de umidade mínima do solo. Os piores resultados foram verificados no manejo de $8 \%$ de umidade do solo, com aplicação de $25 \%$ da dose, independentemente do herbicida, atingindo em média $55 \%$ de controle aos 14 DAA.

Esse fato também foi relatado por Roman et al. (2005): em estudo com aplicação do herbicida carfentrazone-ethyl em Euphorbia heterophylla, a eficiência no controle da planta daninha foi maior (acima de 90\%) nos tratamentos que não se encontravam sob estresse hídrico (potencial de água no solo de -0,002 MPa), enquanto o controle nas plantas submetidas a estresse hídrico $(-0,1 \mathrm{MPa})$ atingiu $78 \%$. De acordo com esses autores, a suscetibilidade de plantas a herbicidas é, muitas vezes, alterada por estresses que afetam a capacidade das plantas em metabolizar os produtos, influenciando o controle das plantas daninhas.

A massa seca das plantas sem aplicação de herbicidas foi maior em comparação à daquelas com aplicação (Figuras 1, 2 e 3). Observou-se redução da massa seca de 40 e $60 \%$ conforme se diminuiu o potencial de água do solo, de -0,03 MPa (manejo hídrico de 13\%) para -0,07 (manejo hídrico de 10\%) e -1,5 (manejo hídrico de 8\%) MPa, respectivamente.

Nas aplicações de 50 e $100 \%$ da dose dos herbicidas, a massa seca das plantas foi semelhante entre os manejos hídricos. Já nas aplicações de $25 \%$ da dose, verificou-se maior distância entre as massas secas, de acordo com o manejo utilizado, sendo mais visivel com a aplicação do herbicida haloxyfop-methyl (Figura 2).

Observou-se que a diferença entre a massa seca de plantas sem aplicação de herbicidas e

Tabela 3 - Porcentagem de controle em plantas de B. plantaginea no estádio de 4- folhas submetidas a diferentes manejos hídricos, sete dias após aplicação de herbicidas. Botucatu-SP, 2008

\begin{tabular}{|c|c|c|c|c|c|}
\hline \multicolumn{2}{|c|}{ Tratamento } & \multicolumn{4}{|c|}{$\%$ da dose de herbicida } \\
\hline Herbicida & Manejo hídrico $(\mathrm{MPa})$ & 0 & 25 & 50 & 100 \\
\hline \multirow{3}{*}{ sethoxydim } & $-0,03$ & 0,00 a C & 21,25 a B & 24,25 a B & 46,25 a A \\
\hline & $-0,07$ & 0,00 a C & $16,25 \mathrm{ab} B$ & $18,25 \mathrm{abc} B$ & 39,25 a A \\
\hline & $-1,50$ & 0,00 a B & $13,50 \mathrm{ab} \mathrm{A}$ & $14,25 \mathrm{abc} A$ & $18,75 \mathrm{~cd} \mathrm{~A}$ \\
\hline \multirow{3}{*}{ haloxyfop-methyl } & $-0,03$ & 0,00 a C & $14,50 \mathrm{ab} \mathrm{B}$ & $21,25 \mathrm{ab} \mathrm{AB}$ & $26,25 \mathrm{bc} \mathrm{A}$ \\
\hline & $-0,07$ & 0,00 a C & $15,00 \mathrm{ab} \mathrm{B}$ & $17,00 \mathrm{abc} \mathrm{AB}$ & $24,50 \mathrm{c} \mathrm{A}$ \\
\hline & $-1,50$ & 0,00 a B & $8,00 \mathrm{~b} \mathrm{AB}$ & $10,00 \mathrm{bc} \mathrm{A}$ & $12,75 \mathrm{dA}$ \\
\hline \multirow{3}{*}{ fluazifop-p-butil } & $-0,03$ & 0,00 a C & 16,25 ab B & 19,50 abc $\mathrm{B}$ & $36,25 \mathrm{ab} \mathrm{A}$ \\
\hline & $-0,07$ & 0,00 a C & $13,25 \mathrm{ab} \mathrm{B}$ & 14,25 abc B & 27,75 bc $\mathrm{A}$ \\
\hline & $-1,50$ & 0,00 a B & 8,75 a $\mathrm{AB}$ & $9,50 \mathrm{c} \mathrm{A}$ & $11,00 \mathrm{dA}$ \\
\hline $\mathrm{F}_{\text {tratamento }}(\mathrm{T})$ & \multicolumn{5}{|c|}{$15,839 * *$} \\
\hline $\mathrm{F}_{\text {dose }}(\mathrm{D})$ & \multicolumn{5}{|c|}{$169,972 * *$} \\
\hline $\mathrm{F}(\mathrm{T}) \mathrm{x}(\mathrm{D})$ & \multicolumn{5}{|c|}{$4,074 * *$} \\
\hline CV $(\%)$ & \multicolumn{5}{|c|}{35,5} \\
\hline
\end{tabular}

Médias seguidas de mesma letra, minúscula na coluna e maiúscula na linha, não diferem entre si pelo teste de Tukey (p $>0,05)$;** Significativo a $1 \%$ de probabilidade. 
Tabela 4 - Porcentagem de controle em plantas de B. plantaginea no estádio de 4-6 folhas submetidas a diferentes manejos hídricos, 14 dias após aplicação de herbicidas. Botucatu-SP, 2008

\begin{tabular}{|c|c|c|c|c|c|}
\hline \multicolumn{2}{|c|}{ Tratamento } & \multicolumn{4}{|c|}{$\%$ da dose de herbicida } \\
\hline Herbicida & Manejo hídrico $(\mathrm{MPa})$ & 0 & 25 & 50 & 100 \\
\hline \multirow{3}{*}{ sethoxydim } & $-0,03$ & 0,00 a C & 82,50 a B & $93,25 \mathrm{a} \mathrm{AB}$ & 100,00 a A \\
\hline & $-0,07$ & 0,00 a C & $76,25 \mathrm{ab} \mathrm{B}$ & 91,25 a $\mathrm{AB}$ & 100,00 a A \\
\hline & $-1,50$ & 0,00 a C & $48,75 \mathrm{c} \mathrm{B}$ & $80,00 \mathrm{ab} \mathrm{A}$ & $85,00 \mathrm{ab} \mathrm{A}$ \\
\hline \multirow{3}{*}{ haloxyfop-methyl } & $-0,03$ & 0,00 a B & 85,00 a A & 96,25 a A & 100,00 a A \\
\hline & $-0,07$ & 0,00 a C & $67,50 \mathrm{abc} \mathrm{B}$ & 89,50 a $\mathrm{A}$ & 99,50 a A \\
\hline & $-1,50$ & 0,00 a C & $58,75 \mathrm{bc} \mathrm{B}$ & $77,50 \mathrm{ab} \mathrm{A}$ & $89,50 \mathrm{Ab} \mathrm{A}$ \\
\hline \multirow{3}{*}{ fluazifop-p-butil } & $-0,03$ & 0,00 a B & 85,00 a A & 91,50 a A & 99,00 a A \\
\hline & $-0,07$ & 0,00 a C & $76,25 \mathrm{ab} B$ & $83,25 \mathrm{ab} A B$ & $97,50 \mathrm{ab} \mathrm{A}$ \\
\hline & $-1,50$ & 0,00 a C & $57,50 \mathrm{bc} \mathrm{B}$ & $65,00 \mathrm{~b} \mathrm{AB}$ & $78,25 \mathrm{~b} \mathrm{~A}$ \\
\hline $\mathrm{F}_{\text {tratamento }}(\mathrm{T})$ & \multicolumn{5}{|c|}{$10,265^{* *}$} \\
\hline $\mathrm{F}_{\text {dose }}(\mathrm{D})$ & \multicolumn{5}{|c|}{$773,641 * *$} \\
\hline $\mathrm{F}(\mathrm{T}) \mathrm{x}(\mathrm{D})$ & \multicolumn{5}{|c|}{$1,891 *$} \\
\hline CV $(\%)$ & \multicolumn{5}{|c|}{14,8} \\
\hline
\end{tabular}

Médias seguidas de mesma letra, minúscula na coluna e maiúscula na linha, não diferem entre si pelo teste de Tukey $(\mathrm{p}>0,05) ; * *$ Significativo a $1 \%$ de probabilidade.

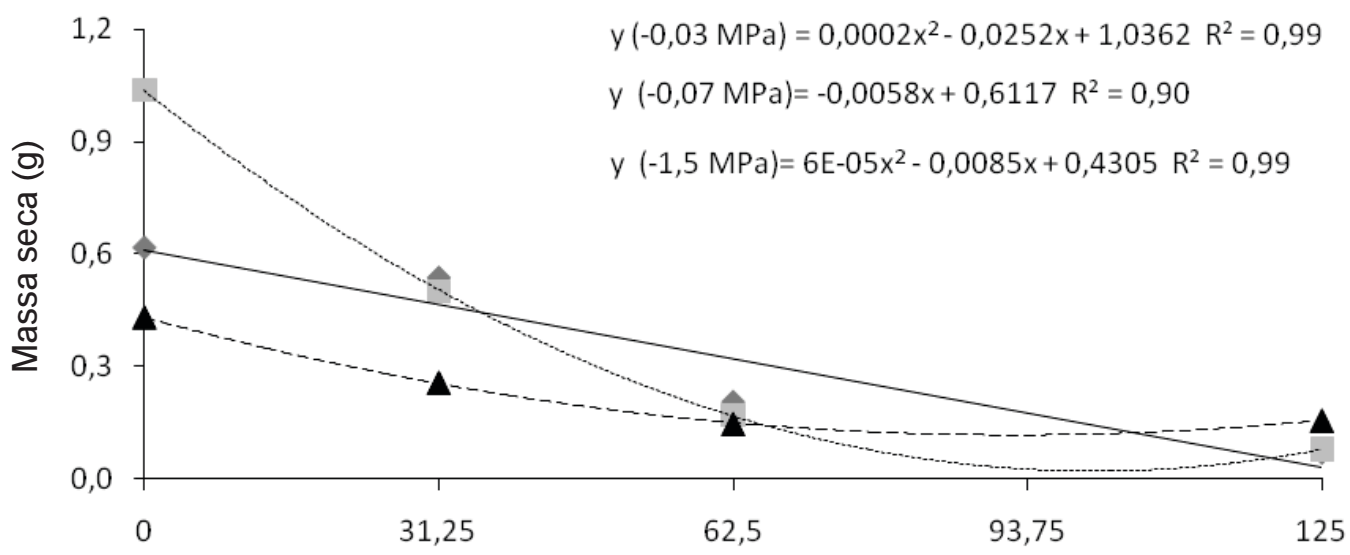

Doses de fluazifop-p-butil (g i.a. ha-1)

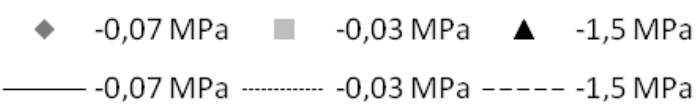

Figura 1 - Massa seca de plantas de B. plantaginea submetidas a diferentes manejos hídricos com aplicação do herbicida fluazifopp-butil, em quatro doses distintas, após 14 dias em plantas no estádio de 4-6 folhas. Botucatu-SP, 2008.

a daquelas com aplicação aumentou de acordo com o incremento da dose aplicada, sendo maior para as plantas sem estresse hídrico (manejo hídrico de $13 \%$ ), em que todos os herbicidas nas aplicações de $100 \%$ da dose causaram, em média, redução de $90 \%$ na massa seca - fato também observado nas plantas mantidas em manejo hídrico de $10 \%$.
Em plantas submetidas a esses mesmos manejos hídricos essa diferença ficou em torno de $70 \%$, com aplicação de $50 \%$ da dose, e $45 \%$ com $25 \%$ da dose. Já a diferença de massa seca entre plantas com e sem aplicação dos produtos, submetidas a estresse hídrico severo (manejo hídrico $8 \%$ ), atingiu em média $60 \%$ com o uso de $100 \%$ da dose e 55 e 30\% com aplicação 
de 50 e $25 \%$ da dose recomendada dos herbicidas, respectivamente.

Pode-se inferir que essa menor diferença entre as massas de plantas com e sem aplicação dos herbicidas, submetidas a manejo hídrico de $8 \%$, está relacionada com a menor eficiência de controle dos produtos sobre elas, proporcionando maiores valores em relação à testemunha sem aplicação.
De acordo com Levene \& Owen (1995), a menor eficiência de controle em plantas submetidas a estresse hídrico pode ser influenciada pelo fato de as folhas apresentarem orientação mais verticalizada do que plantas não estressadas. Essa orientação vertical das folhas poderia reduzir potencialmente a exposição da área foliar e, consequentemente, diminuir a retenção das gotas pulverizadas.
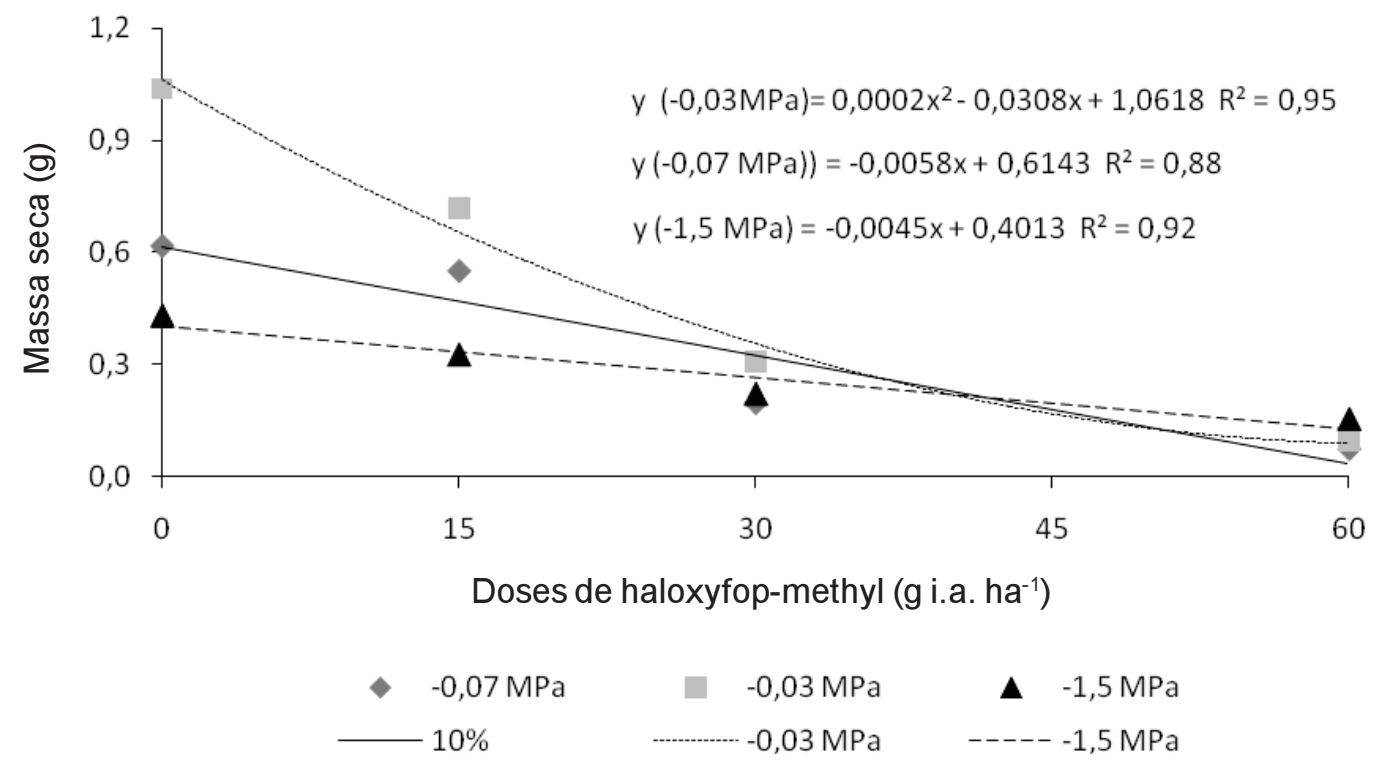

Figura 2 - Massa seca de plantas de B. plantaginea submetidas a diferentes manejos hídricos com aplicação do herbicida haloxyfopmethyl, em quatro doses distintas, após 14 dias em plantas no estádio de 4-6 folhas. Botucatu-SP, 2008.
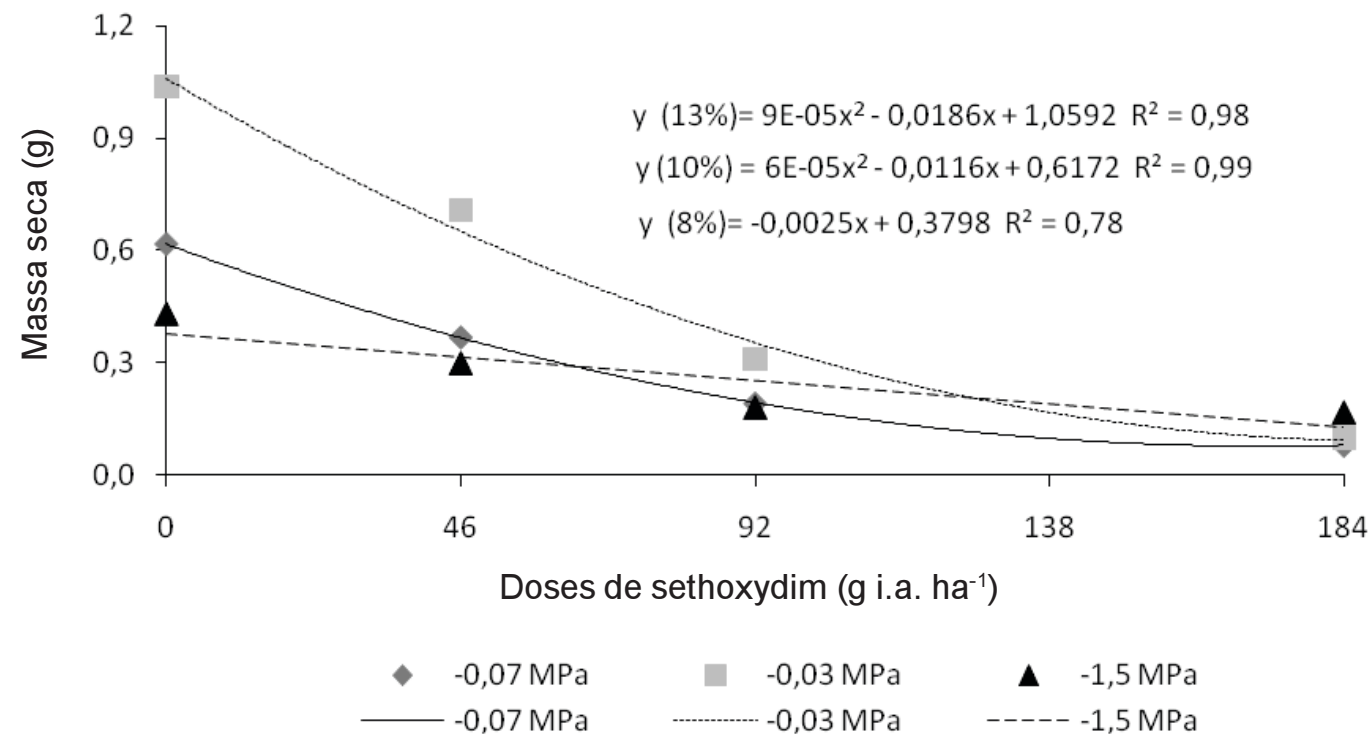

Figura 3 - Massa seca de plantas de B. plantaginea submetidas a diferentes manejos hídricos com aplicação do herbicida sethoxydim, em quatro doses distintas, após 14 dias em plantas no estádio de 4-6 folhas. Botucatu-SP, 2008. 
Nas aplicações sobre plantas no estádio 2 (2-3 perfilhos), com $100 \%$ da dose recomendada, as maiores eficiências de controles, 15 a $20 \%$, foram observadas em plantas sem estresse hídrico (manejo hídrico de 13\%), não havendo diferenças entre os três herbicidas. Observa-se também que não houve diferenças entre as plantas submetidas aos manejos hídricos de 10 e $8 \%$, independentemente do herbicida utilizado, com toxicidade entre $4 \mathrm{e}$ 8\% (Tabela 5).

Com a redução da dose dos herbicidas em $50 \%$, o controle verificado com o uso do haloxyfop-methyl em plantas submetidas a manejo hídrico de $13 \%$ não diferiu quando se aplicaram $100 \%$ da dose. Esse fato repetiu-se em todos os tratamentos com o uso dos manejos hídricos de 10 e $8 \%$. Verificou-se redução de controle de mais de $60 \%$ nos tratamentos com o herbicida fluazifop-p-butil e de $50 \%$ com o sethoxydim em plantas sem estresse hídrico, em comparação com as aplicações de $100 \%$ da dose recomendada. Comportamento semelhante foi observado com o uso de $25 \%$, o qual provocou menor toxicidade em plantas submetidas a manejo hídrico de 10 e $8 \%$, não diferindo das outras doses aplicadas.

Na Tabela 6 são apresentados os resultados de fitointoxicação aos 7 DAA. Com o uso de $100 \%$ da dose dos herbicidas, os resultados provocados pelo haloxyfop-methyl estabilizaram-se em plantas com manejo hídrico de 13\%. Já nas plantas submetidas aos manejos hídricos de 10 e $8 \%$ observou-se aumento acima de $50 \%$ da fitotoxicidade, em comparação com a avaliação aos 3 DAA. Esse aumento também foi observado nas aplicações com os outros herbicidas, porém em menores proporções.

A eficiência de controle obtida com sethoxydim e haloxyfop-methyl em plantas submetidas aos manejos hídricos de 10 e $8 \%$ foi semelhante. Já o comportamento de fluazifopp-butil foi inferior em plantas sob restrição hídrica (manejo hídrico de $8 \%$ ), não atingindo $10 \%$ de controle.

Não se observaram diferenças na eficiência de controle com as reduções da dose, independentemente do herbicida e manejo hídrico utilizado, com exceção do resultado obtido com aplicação de $25 \%$ da dose do herbicida haloxyfo-methyl em plantas sem restrição hídrica (manejo hídrico de 13\%).

Em estudo realizado por Zanatta et al. (2008), a eficácia do teor de água no solo influenciou a eficiência do fomesafen sobre plantas de Amaranthus hybridus, porém o nível de influência variou com a dose aplicada. A

Tabela 5 - Porcentagem de controle em plantas de B. plantaginea no estádio de 2-3 perfilhos submetidas a diferentes manejos hídricos, três dias após aplicação de herbicidas. Botucatu-SP, 2008/2009

\begin{tabular}{|c|c|c|c|c|c|}
\hline \multicolumn{2}{|c|}{ Tratamento } & \multicolumn{4}{|c|}{$\%$ da dose de herbicida } \\
\hline Herbicida & Manejo hídrico (MPa) & 0 & 25 & 50 & 100 \\
\hline \multirow{3}{*}{ sethoxydim } & $-0,03$ & 0,00 a C & 7,25 abc B & 8,75 b B & 15,75 a A \\
\hline & $-0,07$ & $0,00 \mathrm{a} \mathrm{B}$ & $3,75 \mathrm{bc} \mathrm{AB}$ & $6,25 \mathrm{~b} \mathrm{~A}$ & $8,75 \mathrm{~b} \mathrm{~A}$ \\
\hline & $-1,50$ & 0,00 a A & $4,00 \mathrm{bc} \mathrm{A}$ & $4,00 \mathrm{~b} \mathrm{~A}$ & $4,75 \mathrm{~b} \mathrm{~A}$ \\
\hline \multirow{3}{*}{ haloxyfop-methyl } & $-0,03$ & 0,00 a C & 11,25 a B & 20,00 a A & 20,25 a A \\
\hline & $-0,07$ & 0,00 a $\mathrm{A}$ & $4,75 \mathrm{abc} \mathrm{A}$ & $5,25 \mathrm{~b} \mathrm{~A}$ & $5,25 \mathrm{~b} \mathrm{~A}$ \\
\hline & $-1,50$ & 0,00 a $\mathrm{A}$ & $2,25 \mathrm{c} \mathrm{A}$ & $3,25 \mathrm{~b} \mathrm{~A}$ & $4,25 \mathrm{~b} \mathrm{~A}$ \\
\hline \multirow{3}{*}{ fluazifop-p-butil } & $-0,03$ & 0,00 a C & $3,75 \mathrm{bc} \mathrm{BC}$ & $6,25 \mathrm{~b} \mathrm{~B}$ & 20,25 a A \\
\hline & $-0,07$ & 0,00 a B & $9,00 \mathrm{ab} A$ & $9,25 \mathrm{~b} \mathrm{~A}$ & $7,75 \mathrm{~b} \mathrm{~A}$ \\
\hline & $-1,50$ & 0,00 a $\mathrm{A}$ & 3,00 bc $\mathrm{A}$ & $4,00 \mathrm{~b} \mathrm{~A}$ & $5,00 \mathrm{~b} \mathrm{~A}$ \\
\hline $\mathrm{F}_{\text {tratamento }}(\mathrm{T})$ & \multicolumn{5}{|c|}{$20,177 * *$} \\
\hline $\mathrm{F}_{\text {dose }}(\mathrm{D})$ & \multicolumn{5}{|c|}{$75,326^{* *}$} \\
\hline $\mathrm{F}(\mathrm{T}) \times(\mathrm{D})$ & \multicolumn{5}{|c|}{$5,449 * *$} \\
\hline CV $(\%)$ & \multicolumn{5}{|c|}{51,6} \\
\hline
\end{tabular}

Médias seguidas de mesma letra, minúscula na coluna e maiúscula na linha, não diferem entre si pelo teste de Tukey $(\mathrm{p}>0,05)$; ** Significativo a $1 \%$ de probabilidade. 
Tabela 6 - Porcentagem de controle em plantas de B. plantaginea no estádio de 2-3 perfilhos submetidas a diferentes manejos hídricos, sete dias após aplicação de herbicidas. Botucatu-SP, 2008

\begin{tabular}{|c|c|c|c|c|c|}
\hline \multicolumn{2}{|c|}{ Tratamento } & \multicolumn{4}{|c|}{$\%$ da dose de herbicida } \\
\hline Herbicida & Manejo hídrico $(\mathrm{MPa})$ & 0 & 25 & 50 & 100 \\
\hline \multirow{3}{*}{ sethoxydim } & $-0,03$ & 0,00 a B & $17,75 \mathrm{ab} \mathrm{A}$ & 23,25 a A & 26,50 a $\mathrm{A}$ \\
\hline & $-0,07$ & $0,00 \mathrm{a} \mathrm{B}$ & $14,75 \mathrm{abc} \mathrm{A}$ & $20,75 \mathrm{ab} \mathrm{A}$ & $23,75 \mathrm{ab} \mathrm{A}$ \\
\hline & $-1,50$ & 0,00 a B & $11,75 \mathrm{abc} A$ & $15,50 \mathrm{abc} A$ & $17,00 \mathrm{abc} \mathrm{A}$ \\
\hline \multirow{3}{*}{ haloxyfop-methyl } & $-0,03$ & 0,00 a B & $8,00 \mathrm{Bc} \mathrm{B}$ & $19,00 \mathrm{abc} \mathrm{A}$ & $20,00 \mathrm{abc} \mathrm{A}$ \\
\hline & $-0,07$ & 0,00 a B & $11,25 \mathrm{abc} A$ & 12,75 abc $\mathrm{A}$ & $14,50 \mathrm{bc} \mathrm{A}$ \\
\hline & $-1,50$ & $0,00 \mathrm{a} \mathrm{B}$ & $5,25 \mathrm{c} \mathrm{AB}$ & 9,25 bc $\mathrm{AB}$ & $12,25 \mathrm{bc} \mathrm{A}$ \\
\hline \multirow{3}{*}{ fluazifop-p-butil } & $-0,03$ & 0,00 a B & 22,50 a A & $23,00 \mathrm{a} \mathrm{A}$ & 27,00 a A \\
\hline & $-0,07$ & 0,00 a B & $13,00 \mathrm{abc} \mathrm{A}$ & $13,50 \mathrm{abc} \mathrm{A}$ & $13,75 \mathrm{bc} \mathrm{A}$ \\
\hline & $-1,50$ & $0,00 \mathrm{a} \mathrm{A}$ & $5,75 \mathrm{c} \mathrm{A}$ & $8,00 \mathrm{c} \mathrm{A}$ & $9,25 \mathrm{c} \mathrm{A}$ \\
\hline $\mathrm{F}_{\text {tratamento }}(\mathrm{T})$ & \multicolumn{5}{|c|}{$11,024 * *$} \\
\hline $\mathrm{F}_{\text {dose }}(\mathrm{D})$ & \multicolumn{5}{|c|}{$90,448^{* *}$} \\
\hline $\mathrm{F}(\mathrm{T}) \mathrm{x}(\mathrm{D})$ & \multicolumn{5}{|c|}{$1,644 * *$} \\
\hline $\mathrm{CV}(\%)$ & \multicolumn{5}{|c|}{44,2} \\
\hline
\end{tabular}

Médias seguidas de mesma letra, minúscula na coluna e maiúscula na linha, não diferem entre si pelo teste de Tukey $(\mathrm{p}>0,05)$; $* *$ Significativo a $1 \%$ de probabilidade.

eficácia de herbicidas aplicados sobre plantas que se desenvolveram em condições de déficit hídrico foi reduzida, devido à baixa absorção e translocação do produto.

Após 14 dias da aplicação dos produtos, verificou-se controle satisfatório na maioria dos tratamentos - acima de $90 \%$ - com a utilização de $100 \%$ da dose dos herbicidas. Apenas o sethoxydim aplicado em plantas submetidas a estresse hídrico (manejo hídrico de 8\%) obteve controle de $77 \%$ em média; o herbicida fluazifop-p-butil, nas aplicações em plantas submetidas aos manejos hídricos de 10 e 8\%, atingiu eficiência de controle de 87 e $81 \%$, respectivamente.

$\mathrm{Na}$ aplicação de herbicidas diretamente sobre as plantas daninhas (pós-emergência), para uma eficiente ação do herbicida, é importante que essas plantas não estejam em estresse hídrico e sim com as folhas túrgidas (Blanco, 2009).

Nas aplicações de 50\% da dose dos herbicidas, também se notou controle satisfatório: em média, $80 \%$. O melhor resultado (acima de $90 \%$ ) foi obtido com a aplicação do fluazifopp-butil em plantas com manejo hídrico de $13 \%$. Os menores controles são observados em plantas sob estresse hídrico (manejo hídrico de $8 \%$ ).
A redução da dose recomendada dos herbicidas em $25 \%$ prejudicou muito os resultados de controle, atingindo em média $40 \%$, independentemente do manejo hídrico e do herbicida, com exceção das aplicações com haloxyfopmethyl e fluazifop-p-butil em plantas sem estresse hídrico (manejo hídrico de $13 \%$ ), atingindo 60 e $74 \%$ de eficiência de controle, respectivamente.

De acordo com Procópio et al. (2001), em estudo com aplicação de s-metolachlor em $B$. plantaginea, a dose recomendada pela empresa fabricante $\left(0,96 \mathrm{~kg} \mathrm{ha}^{-1}\right)$ resultou em controle de $86 \%$ em área irrigada antes da aplicação e de $78 \%$ com irrigação após aplicação; com redução da dose em $50 \%$, o controle foi de $77 \%$ em área irrigada e de $67 \%$ em área sem irrigação, aos 20 DAA.

Em aplicação com o herbicida atrazine em plantas de $B$. plantaginea, Maciel et al. (2002) constataram que aos 8 DAA a absorção do herbicida proporcionou controle satisfatório $(85,0$ e $85,7 \%)$ da infestante no estádio de duas a três folhas, independentemente da condição de umidade do solo. Entretanto, para plantas no estádio de quatro a cinco folhas, a absorção foliar de atrazine proporcionou controle insatisfatório aos 8 e 16 DAA ( $\leq 42,5 \%)$, sendo esse efeito ainda mais evidente quando o herbicida foi aplicado em solo seco. 
As massas secas de plantas após 14 dias que receberam aplicação dos produtos no estádio de 2-3 perfilhos estão representadas nas Figuras 4, 5 e 6. Observou-se maior massa daquelas sem estresse hídrico (manejo hídrico de $13 \%$ ) em comparação às com manejos hídricos de 10 e $8 \%$, sendo verificadas diferenças entre elas de $46 \%$, em média; a diferença entre as massas de plantas com manejos hídricos de 10 e $8 \%$ foi de $24 \%$.
A redução da dose de aplicação de todos os herbicidas não influenciou a massa seca de plantas mantidas em manejo hídrico de $8 \%$, fazendo assim que esta atingisse maiores valores com a aplicação de $100 \%$ da dose, independentemente do produto utilizado. Evidencia-se, assim, a menor fitointoxicação causada pelos herbicidas em plantas submetidas a estresse.

Tabela 7 - Porcentagem de controle em plantas de B. plantaginea no estádio de 2-3 perfilhos submetidas a diferentes manejos hídricos, 14 dias após aplicação de herbicidas. Botucatu-SP, 2008

\begin{tabular}{|c|c|c|c|c|c|}
\hline \multicolumn{2}{|c|}{ Tratamento } & \multicolumn{4}{|c|}{$\%$ da dose de herbicida } \\
\hline Herbicida & Manejo hídrico $(\mathrm{MPa})$ & 0 & 25 & 50 & 100 \\
\hline \multirow{3}{*}{ sethoxydim } & $-0,03$ & 0,00 a D & $35,75 \mathrm{C} \mathrm{C}$ & $83,75 \mathrm{ab} B$ & 100,00 a A \\
\hline & $-0,07$ & 0,00 a C & $33,75 \mathrm{C} \mathrm{B}$ & $79,50 \mathrm{ab} A$ & $92,50 \mathrm{ab} \mathrm{A}$ \\
\hline & $-1,50$ & 0,00 a C & $30,50 \mathrm{C} \mathrm{B}$ & $72,50 \mathrm{~b} \mathrm{~A}$ & $77,50 \mathrm{~b} \mathrm{~A}$ \\
\hline \multirow{3}{*}{ haloxyfop-methyl } & $-0,03$ & 0,00 a $\mathrm{C}$ & $61,25 \mathrm{~A} \mathrm{~B}$ & $85,00 \mathrm{ab} A$ & 100,00 a A \\
\hline & $-0,07$ & 0,00 a C & $57,50 \mathrm{ab} \mathrm{B}$ & $85,75 \mathrm{ab} \mathrm{A}$ & $95,00 \mathrm{ab} \mathrm{A}$ \\
\hline & $-1,50$ & 0,00 a C & 38,75 bc B & 43,75 c B & $92,00 \mathrm{ab} \mathrm{A}$ \\
\hline \multirow{3}{*}{ fluazifop-p-butil } & $-0,03$ & 0,00 a $\mathrm{C}$ & $74,00 \mathrm{~A} \mathrm{~B}$ & 93,75 a $\mathrm{AB}$ & $94,00 \mathrm{ab} \mathrm{A}$ \\
\hline & $-0,07$ & 0,00 a $\mathrm{C}$ & $55,75 \mathrm{ab} \mathrm{B}$ & $88,25 \mathrm{ab} A$ & $87,00 \mathrm{ab} A$ \\
\hline & $-1,50$ & 0,00 a C & $27,50 \mathrm{C} \mathrm{B}$ & 30,75 c B & $81,25 \mathrm{ab} A$ \\
\hline $\mathrm{F}_{\text {tratamento }}(\mathrm{T})$ & \multicolumn{5}{|c|}{$20,533 * *$} \\
\hline $\mathrm{F}_{\text {dose }}(\mathrm{D})$ & \multicolumn{5}{|c|}{$753,958 * *$} \\
\hline $\mathrm{F}(\mathrm{T}) \times(\mathrm{D})$ & \multicolumn{5}{|c|}{$7,285 * *$} \\
\hline $\mathrm{CV}(\%)$ & \multicolumn{5}{|c|}{16,5} \\
\hline
\end{tabular}

Médias seguidas de mesma letra, minúscula na coluna e maiúscula na linha, não diferem entre si pelo teste de Tukey $(\mathrm{p}>0,05) ;{ }^{* *}$ Significativo a $1 \%$ de probabilidade.

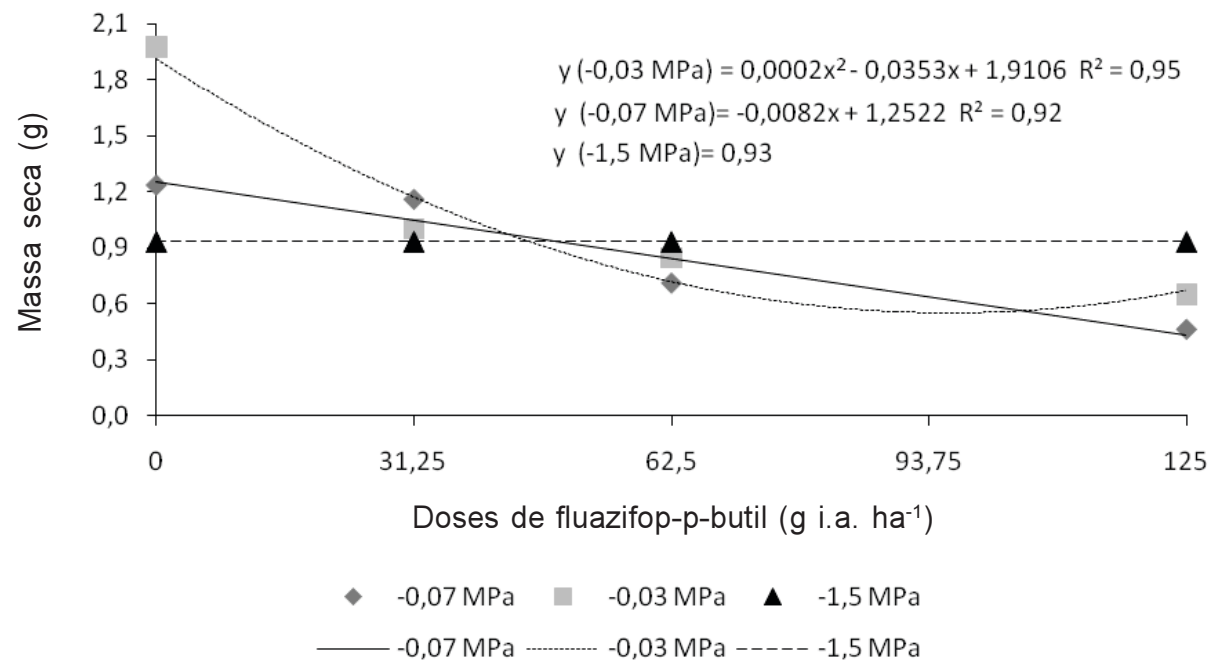

Figura 4 - Massa seca de plantas de B. plantaginea submetidas a diferentes manejos hídricos com aplicação do herbicida fluazifop-p-butil, em quatro doses distintas, após 14 dias em plantas no estádio de 2-3 perfilhos. Botucatu-SP, 2008. 


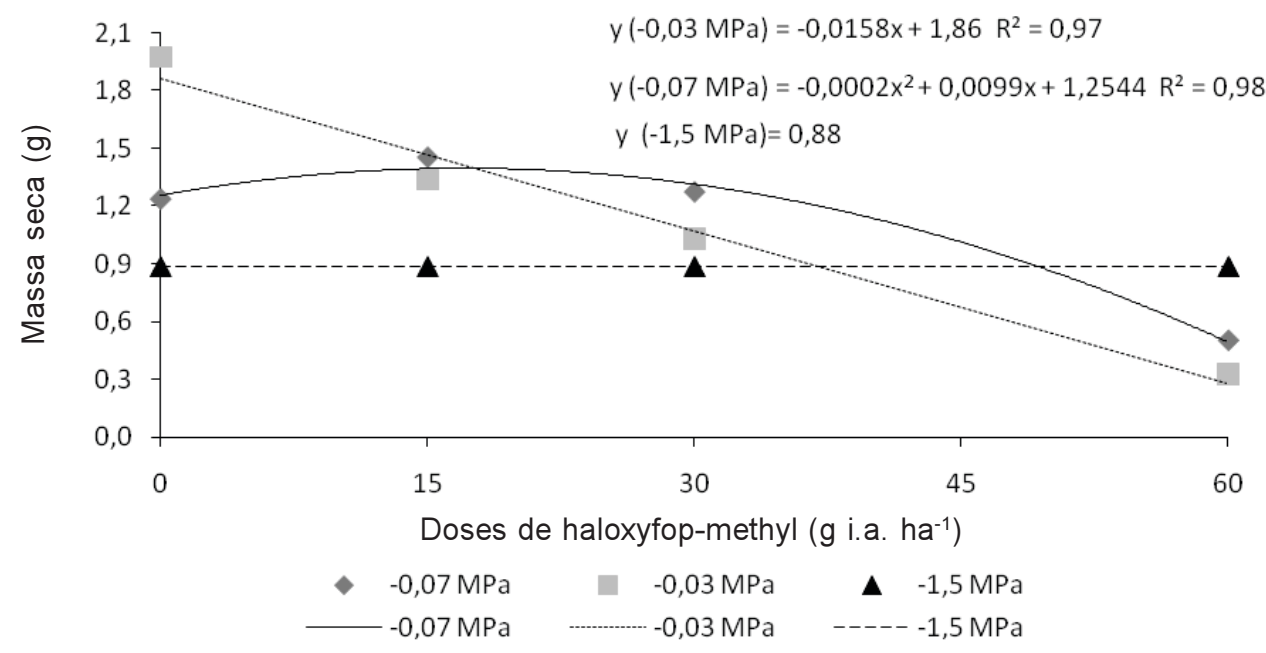

Figura 5 - Massa seca de plantas de B. plantaginea submetidas a diferentes manejos hídricos com aplicação do herbicida haloxyfopmethyl, em quatro doses distintas, após 14 dias em plantas no estádio de 2-3 perfilhos. Botucatu-SP, 2008.

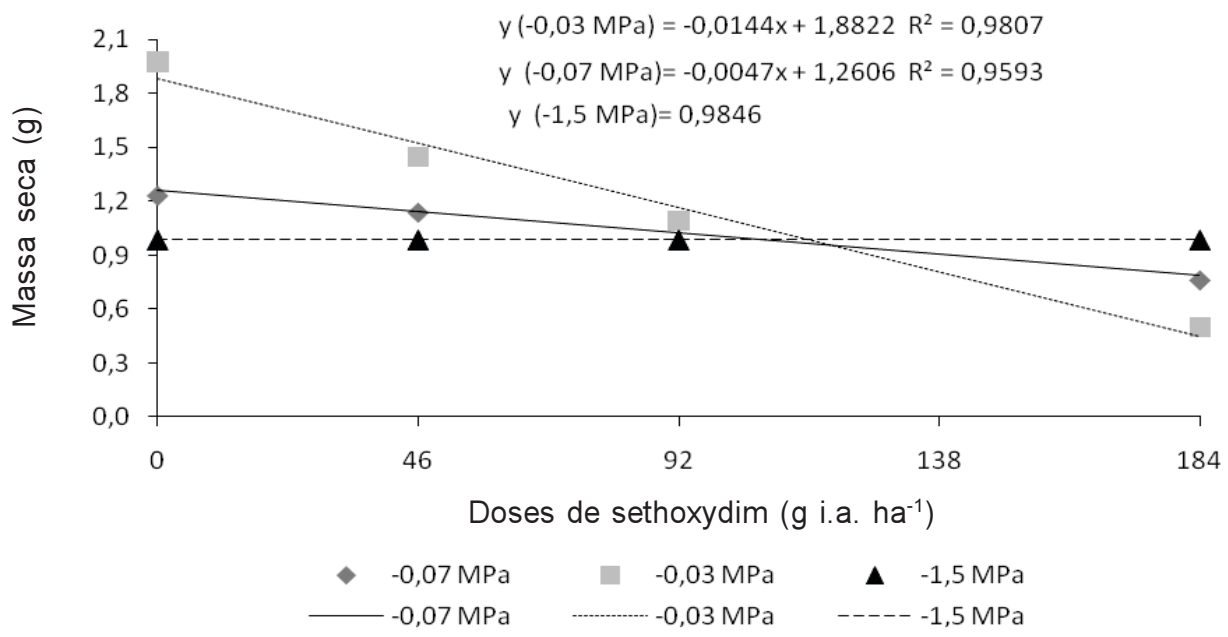

Figura 6 - Massa seca de plantas de B. plantaginea submetidas a diferentes manejos hídricos com aplicação do herbicida sethoxydim, em quatro doses distintas, após 14 dias em plantas no estádio de 2-3 perfilhos. Botucatu-SP, 2008.

Com a aplicação do herbicida sethoxydim (Figura 6), pode-se observar que entre as massas de plantas com os diferentes manejos hídricos, com a aplicação de $25 \%$ da dose, os valores de massa seca acompanharam o mesmo comportamento quando estas estavam sem aplicação do produto, igualando seus valores com o uso de $50 \%$ da dose e invertendo com o uso de $100 \%$, a qual resultou em menores valores de massa em plantas sem estresse hídrico (manejo hídrico de 13\%) e maiores em plantas submetidas a estresse severo (manejo hídrico de $8 \%$ ).
Nas condições em que o presente trabalho foi realizado, pode-se concluir que a eficiência de controle foi menor em plantas mantidas no potencial de água no solo de -1,5 MPa (manejo hídrico de $8 \%$ ), independentemente do herbicida utilizado, nos dois estádios de aplicação, com exceção do haloxyfop-methyl aplicado no estádio 2; os melhores controles foram observados em plantas submetidas aos manejos hídricos de 13 e 10\% e com aplicação dos herbicidas sethoxydim e haloxyfop-methyl (controle acima de $90 \%$ ) nos dois estádios de crescimento das plantas. 
Não houve diferenças de controle entre as aplicações com $100 \%$ da dose recomendada e o fracionamento desta em $50 \%$, com exceção das plantas mantidas em manejo hídrico de $8 \%$ e aplicação no estádio 2 . No entanto, a eficiência dos herbicidas foi reduzida com o uso de $25 \%$ da dose recomendada.

\section{LITERATURA CITADA}

ABBOTT, L. B.; STERLING, T. M. Recovery of African rue seedlings from water stress: Implications for recruitment and invasion. African rue (Peganum harmala) seedling response to herbicides applied under water-deficit stress. Weed Sci., v. 54 , p. $198-204,2006$

BLANCO, F. M. G. Controle de plantas daninhas em batata. São Paulo: Instituto Biológico, [200-]. Disponível em: $<\mathrm{http}$ //www.biologico.sp.gov.br/artigos_ok.php?id_artigo $=57>$. Acesso em: 15 out 2009 .

BOYDSTON, R. A. Soil water content affects the activity of four herbicides on green foxtail. Weed Sci., v. 38, p. 578-582, 1990.

DEUBERT, R. Ciências das plantas daninhas: fundamentos. Jaboticabal: FUNEP, 1992. v. 1. 431 p.

DICKSON, R. L. et al. Effect of water stress nitrogen and gibberellic acid on fluazifop and glyphosate activity on oats (Avena sativa). Weed Sci., v. 38, p. 54-61, 1990.

FLECK, N. G.; CUNHA, M. M.; VARGAS, L. Dose reduzida de clethodim no controle de papuã na cultura da soja, em função da época de aplicação. Planta Daninha, v. 15, n. 1, p. 18-24, 1997

KELLS, J. J.; MEGGIT, W. F.; PENNER, D. Absorption, translocation, and activity of fluazifop-P-butyl as influenced by plant growth stage and environment. Weed Sci., v. 32, p. $143-149,1984$.

KISSMANN, K. G. Plantas infestantes e nocivas. 2.ed. São Paulo: Basf Brasileira, 1997. p. 415-420.

KLAR, A. E. Evapotranspiração. In: KLAR, A. E. A água no sistema solo-planta-atmosfera. 2.ed. São Paulo: Nobel, 1984. $408 \mathrm{p}$.

LEVENE, B. C.; OWEN, M. D. K. Effect of moisture stress and leaf age on bentazon absorption in common cocklebur (Xanthium strumarium) and velvetleaf (Abutilon theophrasti). Weed Sci., v. 43, n. 1, p. 7-12, 1995.
LORENZI, H. Plantas daninhas do Brasil: terrestres, aquáticas, parasitas e tóxicas. 3.ed. Nova Odessa: Plantarum, 2000. 624 p.

MACIEL, C. D. G. et al. Método alternativo de avaliação da absorção de atrazine por plantas de Brachiaria plantaginea.

Planta Daninha, v. 20, n. 3, p. 431-438, 2002.

PEREGOY, R. et al. Moisture stress effects on the absorption, translocation, and metabolism of haloxyfop in johnsongrass (Sorghum halepense) and large crabgrass (Digitaria sanguinalis). Weed Sci., v. 38, p. 331-337, 1990.

PIRES, J. L. F. et al. Redução na dose do herbicida aplicado em pós-emergência associada a espaçamento reduzido da cultura de soja para controle de Brachiaria plantaginea. Planta Daninha, v. 19, n. 3, p. 337-343, 2001.

PROCÓPIO, S. O. et al. Eficiência do s-metolachlor no controle de Brachiaria plantaginea na cultura do feijão sob dois manejos de irrigação. Planta Daninha, v. 19, n. 3, p. $427-433,2001$.

RODRIGUES, J. D. Absorção, translocação e modo de ação de defensivos. Botucatu: UNESP, Instituto de Biociências, 1994. 106 p.

ROMAN, E. S.; VARGAS, L.; RIBEIRO, M. C. F. Efeito do teor de umidade do solo na seletividade e na eficiência de carfentrazone-ethyl no controle de plantas daninhas na cultura da soja. R. Bras. Herbic., 2005. Disponível em: $<$ http:// www.upf.br/rbherbicidas/download/RBH_Carfentrazone.pdf >. Acesso em: 19 out. 2009.

SOCIEDADE BRASILEIRA DA CIÊNCIA DAS PLANTAS DANINHAS - SBCPD. Procedimentos para instalação, avaliação e analise de experimentos com herbicidas. Londrina: $1995.42 \mathrm{p}$.

SPACER, V.; VIDAL, R. A. Eficácia de herbicidas graminicidas aplicados em pré-emergência no sistema de semeadura direta do milho. Planta Daninha, v. 18, n. 2, p. 373-380, 2000.

XIE, H. S. et al. Influence of water stress on absorption, translocation and phytotoxicity of fenozaprop-ethyl and imazamethabenz-methyl in Avena fatua. Weed Res., v. 36, p. $65-71,1996$

WALDECKER, M. A.; WYSE, D. L. Soil moisture effects on glyphosate absorption and translocation in common milkweed (Asclepias syriaca). Weed Sci., v. 33, p. 299-305, 1985.

ZANATTA, J. F. et al. Teores de água no solo e eficácia do herbicida fomesafen no controle de Amaranthus hybridus. Planta Daninha, v. 26, n. 1, p. 143-155, 2008. 